\title{
The Influence of ABS with Different Contents of Butadiene on the Crystallization Behavior of MCPA6/ABS Copolymer Alloys
}

\author{
Bo-Fen HUANG ${ }^{1, a,{ }^{*},}$ Xiang LIANG ${ }^{2, b}$, Xiao LI ${ }^{3, c}$, Dan LI,
}

${ }^{1}$ School of Materials Science and Engineering, Nanchang University, Nanchang, China

${ }^{2}$ Jiangxi University of Traditional Chinese Medicine, Nanchang, China

${ }^{3}$ Hefei Genius Advanced Material Co., Ltd, Hefei, China

${ }^{4}$ National engineering technology research center for LED on Si substrate, Nanchang, China

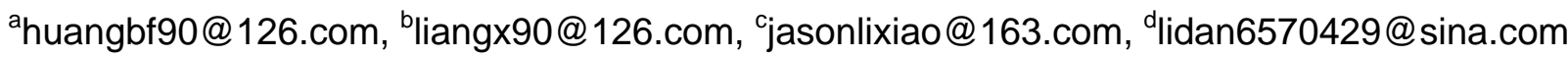

${ }^{*}$ Corresponding author

Keywords: Monomer Casting Polyamide 6 (MCPA6), ABS, Copolymer Alloys, Butadiene Content, Crystallization.

\begin{abstract}
Two kinds of Acrylonitrile-Butadiene-Styrene (ABS) resins (ABS749S and ABS757) were manufactured as MCPA6/ABS copolymer alloys by in situ polymerization and their crystallization properties were analyzed by Differential Scanning Calorimetry (DSC). The DSC results indicated that $\mathrm{ABS}$ did not change coexistence state of the two polymorph existed in the MCPA6, while it inhibited the MCPA6's crystallization ability. Melting enthalpy, crystallization temperature and crystallinity of MCPA6/ABS dropped with the increase of the ABS. When the content of the ABS was the same, the difficulty of the molecular chains' movement and the nucleation process was raised with the increase of the butadiene soft segment's content. The crystallization degree of ABS757 was larger than that of ABS749S, which were respectively $18.1 \%$ and $16.5 \%$.
\end{abstract}

\section{Introduction}

Monomer Casting Polyamide 6 (MCPA6) owns great mechanical properties, good self-lubricating and small noise damping properties, which is widely used in manufacturing large products such as gears, bearings and sliders ${ }^{[1-3]}$. However, it has some defects such as low impact strength and the MCPA6 products have poor creep resistance and dimensional stability after absorbing water. Therefore, more and more attention is focus on modification of MCPA $6^{[4]}$.

ABS resin is a terpolymer consisted by acrylonitrile (A), butadiene (B) and styrene (S). By mixing it with another material, the soft segment of the butadiene provides a good shock resistance for the new compound, while the acrylonitrile provides processing fluidity ${ }^{[5]}$. Simultaneously, its strength corrosion resisting is enhanced due to both surface smoothness and the acrylonitrile enhance. As a result, the new material usually owns a better performance. ABS can modify the MCPA6's stiffness and mechanical properties because of the reaction between nitrile with caprolactam. In this paper, the reactions between MCPA6 and ABS with different marks are analyzed, as well as the influence of the butadiene content on the MCPA6/ABS alloys' mechanical properties.

\section{Experimental}

\section{Main Materials}

Caprolactam (CL): AR, Ludwigshafen, Germany Company. Acrylonitrile - butadiene - styrene (ABS): 749S (18\% butadiene) and 757 (10\% butadiene), Zhenjiang Chi Mei Corporation. 2, 4-toluene diisocyanate (TDI): AR, Shanghai chemical reagent Co., Ltd. 


\section{Preparation of MCPA6/ABS Copolymer Alloys}

An amount of caprolactam was added into a three-entrance glass bottle and melt with the protection of nitrogen. The melt powder is then respectively mixed with different marks and mass ratio ABS particles until all of them were resolved, and the mixed solution became transparent. Then solution was processed by vacuumed distillation for a period of time. Next, by keeping the vacuum degree between 0.005 and 0.010 , sodium hydroxide was added with the continuous vacuumed distillation. A certain amount of TDI was added to the bottle and mixed uniformly, and the mixed TDI was poured into a mold preheated to $145^{\circ} \mathrm{C}$, keeping the temperature for 40 minutes. MCPA6/ABS alloys were obtained by demolding at the low temperature.

\section{Measurements}

The melting and crystallization behaviors of the samples were investigated by a Parkin - Elmer Diamond differential scanning calorimeter (DSC). The samples of about $5 \mathrm{mg}$ were heated at $250^{\circ} \mathrm{C}$ with $80^{\circ} \mathrm{C} / \mathrm{min}$ and keeping for 2 minutes in order to cancel the thermal history. The sample was then cooled to $70^{\circ} \mathrm{C}$ with cooling rate of $20^{\circ} \mathrm{C} / \mathrm{min}$, then with a heating rate of $20^{\circ} \mathrm{C} / \mathrm{min}$ heating to $240^{\circ} \mathrm{C}$; respectively recording process during heating and cooling.

\section{Results and Discussion}

DSC was used to conduct the isothermal circulation of MCPA6 and three different marks ABS alloys, recording their second warming and cooling curves and relevant crystal parameters were induced into the table.

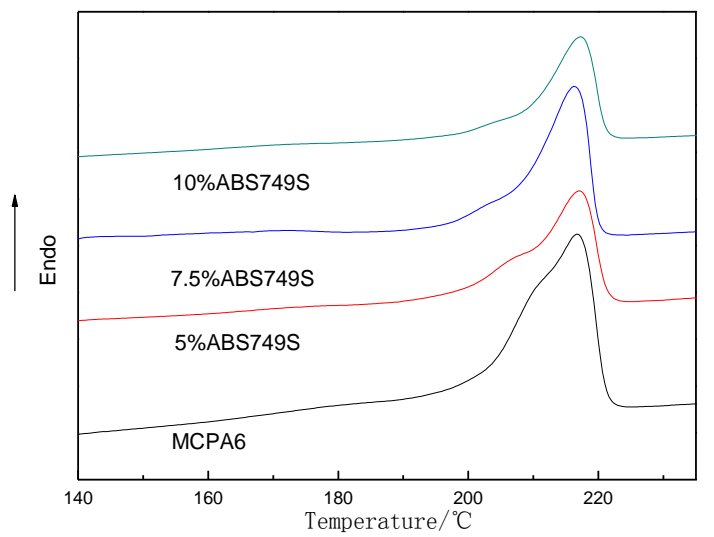

Fig.1 DSC Second Heating Curves of MCPA6 and MCPA6/ABS749S Copolymer Alloys

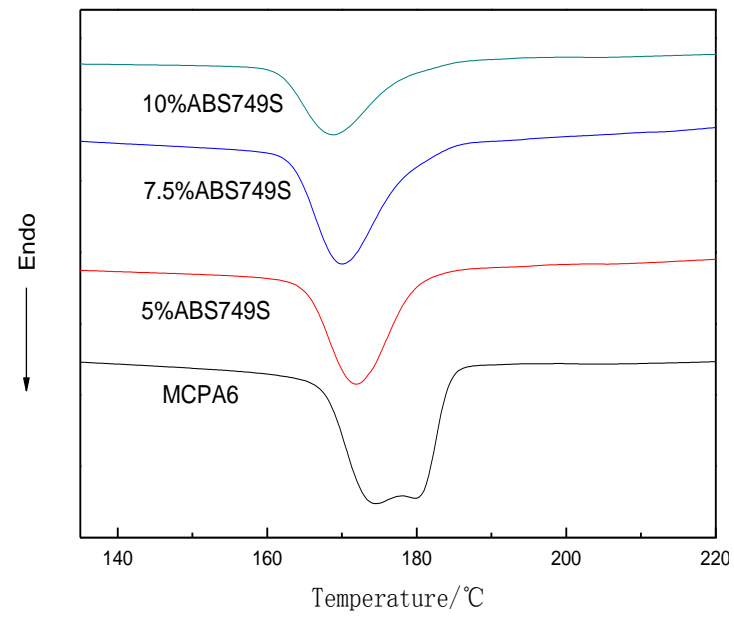

Fig.2 DSC Cooling Curves of MCPA6 and MCPA6/ABS749S Copolymer Alloys 
According to the figure 1 , the melting temperature of the MCPA6 was $216.6^{\circ} \mathrm{C}$. With the involvement of the ABS749S, the melting peak moved to the low temperature zone. When its contents were $10 \%$, the temperature reached the minimum value, which was $215^{\circ} \mathrm{C}$, and the melting peak gradually stepped down and broad. On the one hand, this was due to the nitrile grouping attended into the in situ polymerization, leading to the growth of the ABS-co-MCPA6 in the MCPA6/ABS copolymer system and impeding the MCPA6's crystallization. Therefore, the melting peak stepped down compared with the MCPA6's heating curve. On the other hand, with the modification of the interphase compatibility, the energy for the growth of the MCPA6 spherical crystal gradually increased as well, leading to the imperfect of the crystallization that the amorphous component tend to grow. The imperfect crystal existed in the polymers melted in the low temperature zone, while the perfect crystal melted in the high temperature zone. Consequently, the copolymer alloys' melting peak gradually reduced compared with the increasing MCPA6's heating curve.

Figure 2 illustrates the copolymer alloy's cooling crystallization curve. It indicated that the MCPA6 crystallization peak was $176.7^{\circ} \mathrm{C}$. With ABS749's addition, the position of the melting peak deviated towards the low temperature zone. Among them, the lowest temperature was $167.2^{\circ} \mathrm{C}$ with 10\% ABS749S. The reason for this phenomenon was probably because the existence of the ABS molecular chain which disturbs the order of MCPA6 chains by impeding the molecular chains' move and lower their recrystallization. Consequently, the temperature for the copolymer's crystallization tended to decrease. Simultaneously, although ABS749S increased the difficulty of copolymer alloys' recrystallization, the crystal behaviors were not changed. Therefore, the creation of the crystal was not affect by it. The collection curve was illustrated in the table below.

Table 1 reflects the influence of ABS749S on the MCPA6's properties. The fusion enthalpy during the secondary heating process and the crystallization temperature during the cooling process showed a trend of decrease. Through formula (2), the MCPA6 and its alloy's crystalline could be figured out.

Tab.1 Melting and Crystallization Parameters of MCPA6/ABS749S Copolymer Alloys

\begin{tabular}{|c|c|c|c|c|c|c|c|}
\hline \multirow{2}{*}{ No. } & \multirow{2}{*}{$\begin{array}{c}\text { ABS749S } \\
(\mathrm{wt} \%)\end{array}$} & \multicolumn{3}{|c|}{ Second Heating } & \multicolumn{3}{c|}{ Cooling } \\
\cline { 3 - 8 } & $\left({ }^{\circ} \mathrm{C}\right)$ & $\begin{array}{c}\Delta \mathrm{Hf} \\
(\mathrm{J} / \mathrm{g})\end{array}$ & $\begin{array}{c}\text { Xc (DSC) } \\
(\%)\end{array}$ & $\begin{array}{c}\Delta \mathrm{Hc} \\
(\mathrm{J} / \mathrm{g})\end{array}$ & $\begin{array}{c}\mathrm{Tc}, \mathrm{m} \\
\left({ }^{\circ} \mathrm{C}\right)\end{array}$ & $\begin{array}{c}\Delta \mathrm{Tc} \\
\left({ }^{\circ} \mathrm{C}\right)\end{array}$ \\
\hline 1 & 0 & 216.6 & 38.7 & 20.3 & 44.0 & 176.7 & 39.9 \\
\hline 2 & 5 & 216.1 & 35.3 & 18.6 & 40.3 & 169.3 & 46.8 \\
\hline 3 & 7.5 & 215.8 & 32.9 & 17.3 & 37.5 & 168.5 & 47.3 \\
\hline 4 & 10 & 215.0 & 31.4 & 16.5 & 35.7 & 167.2 & 47.8 \\
\hline
\end{tabular}

Note: Tm: melting peak temperature; $\Delta \mathrm{Hf}$ : melting enthalpy; Xc: degree of crystallinity; $\Delta \mathrm{Hc:}$ crystallization enthalpy; Tc, m: peak crystallization temperature; $\Delta \mathrm{Tc}$ : undercooling.

$$
X c_{(D S C)}=\frac{\Delta H_{f}}{\Delta H_{\gamma e f}}
$$

From the table 1, it could be concluded that the crystalline decreases due to the ABS749S. When the content was $10 \%$, the crystalline reached the bottom value, which was $16.5 \%$, showing a $18.7 \%$ decline. Comparing with the mechanical alloying, the ABS749S nitrile grouping in the in situ polymerization could considerably modify the interphase compatibility, which could enhance the dispersity of the whole system and enlarge the molecular chains' steric hindrance; new polar grouping changed the structure and arrangement of the hydrogen bond, further causing the difficulty in the move of the molecular chains. Consequently, the copolymer alloy's crystallization became much tougher than the pure MCPA6, leading to the decrease of the crystalline. 


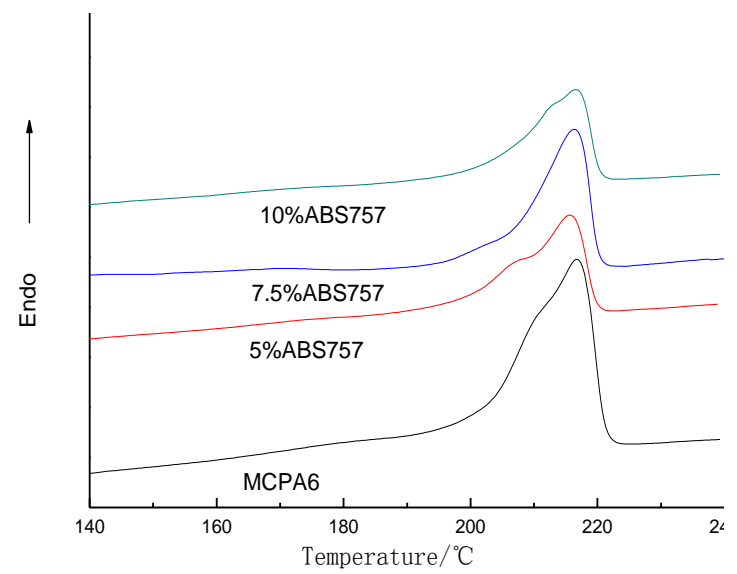

Fig.3 DSC Second Heating Curves of MCPA6 and MCPA6/ABS757 Copolymer Alloys

Figure 3 shows the copolymer's secondary heating curve. It indicates that ABS757 made the melting peak deviates towards the low temperature zone. The crystallization temperature decreased from the $216.6^{\circ} \mathrm{C}$ to $215.6^{\circ} \mathrm{C}$ with the gradually narrowing melting peak.

Figure 4 indicates that the crystallization peak deviates towards the low temperature zone and the crystallization temperature gradually decreases from $176.7^{\circ} \mathrm{C}$ to $168.9^{\circ} \mathrm{C}$ with the decrease of the intense of the crystal peak. The relevant parameters of the MCPA6/ABS757 are shown in the table 2 .

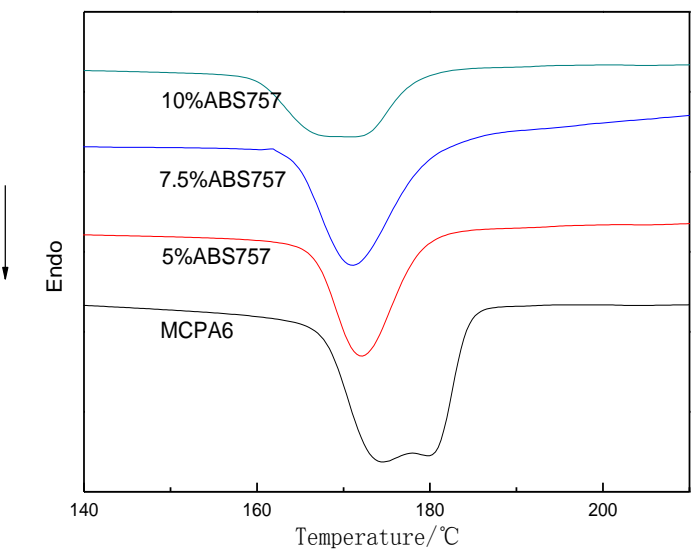

Fig.4 DSC Cooling Curves of MCPA6 and MCPA6/ABS757 Copolymer Alloys

Tab.2 Melting and Crystallization Parameters of MCPA6/ABS757 Copolymer Alloys

\begin{tabular}{|c|c|c|c|c|c|c|c|}
\hline \multirow{2}{*}{ No. } & \multirow{2}{*}{$\begin{array}{c}\text { ABS757 } \\
(\mathrm{wt} \%)\end{array}$} & $\begin{array}{c}\mathrm{Tm} \\
\left({ }^{\circ} \mathrm{C}\right)\end{array}$ & $\begin{array}{c}\Delta \mathrm{Hf} \\
(\mathrm{J} / \mathrm{g})\end{array}$ & $\begin{array}{c}\text { Xc (DSC) } \\
(\%)\end{array}$ & $\begin{array}{c}\Delta \mathrm{Hc} \\
(\mathrm{J} / \mathrm{g})\end{array}$ & $\begin{array}{c}\mathrm{Tc}, \mathrm{m} \\
\left({ }^{\circ} \mathrm{C}\right)\end{array}$ & $\begin{array}{c}\Delta \mathrm{Tc} \\
\left({ }^{\circ} \mathrm{C}\right)\end{array}$ \\
\hline 1 & 0 & 216.6 & 38.7 & 20.3 & 44.0 & 176.7 & 39.9 \\
\hline 2 & 5 & 216.2 & 36.7 & 19.3 & 41.8 & 172.1 & 44.1 \\
\hline 3 & 7.5 & 215.9 & 35.9 & 18.9 & 41.3 & 169.6 & 46.3 \\
\hline 4 & 10 & 215.6 & 34.4 & 18.1 & 39.9 & 168.9 & 46.7 \\
\hline
\end{tabular}

Table 2 illustrates the each crystallization parameter for the MCPA6/ABS757 copolymers. To varying degrees, ABS757 changed MCPA6's every crystallization parameter that all the melting temperature during the secondary heating process, Melting entropy as well as crystallization 
temperature during the cooling process and crystallization degree reduced. When the content of the ABS757 was $10 \%$, the value reached the bottom (18.1\%), having a $10.8 \%$ decrease compared to the MCPA6.

According to the table 1 and 2, with the increase of the content of the soft segment of the butadiene, the crystallization degree of the copolymer alloys gradually decreased from the ABS757 to the ABS749S. When it is $5 \%$, the crystallization degrees were respectively $19.3 \%$ and $18.6 \%$; when $7.5 \%$, they were respectively $18.9 \%$ and $17.3 \%$; when $10 \%$, they were merely $18.1 \%$ and $16.5 \%$. The reason for that was because when the content of the ABS was the same, the move and the nucleation of the copolymer chains became increasingly difficult with the increase of the soft segment of the butadiene. Therefore, the difficulty of the copolymer crystallization reduced with the increase of the content of the butadiene.

\section{Conclusions}

In-situ polymerization of caprolactam anion was used to prepare MCPA6/ABS polymer alloy, and crystalline properties of the alloy were studied. Analysis showed that ABS content affected the crystallization behavior of MCPA6 crystallizability and reduced its degree of crystallinity, but did not change its original crystallization behavior. With the increase of ABS content, melting enthalpy, crystallization temperature and degree of crystallinity of MCPA6/ABS alloy gradually decreased. When the ABS contents were the same, the degree of crystallinity gradually decreased from ABS757 to ABS749S due to increase content of soft segment butadiene polymer alloy, which were respectively $18.1 \%$ and $16.5 \%$.

\section{References}

[1] Bofen Huang, Hanxuan Liang and Yanchao Bao. Applied Mechanics and Materials, 426 (2013) 295-298.

[2] S. George, K. T. Varughese, S. Thomas. Polymer, 41(2000) 5485-5503.

[3] Z. N. Yin, T. J. Wang. Materials Science and Engineering, 527(2010) 1461-1468.

[4] R. Balart, J. Lopez, D. Garcia, M. Dolores Salvador. European Polymer Journal, 41(2005) 2150-2160.

[5] Bofen Huang, Dan Li and Zhiyuan Li. Journal of Applied Polymer Science, 122(2011) 586. 